Uluslararası Mühendislik

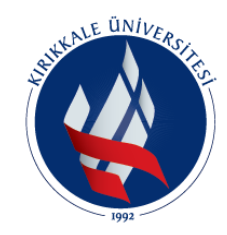

\title{
Kriyojenik Hidrojen Yakıtlı 100 kN İtki Kapasiteli Bir Roketin RPA Programı ile Kavramsal Tasarım ve Analizi
}

\section{Conceptual Design and Analysis of a Cryogenic Hydrogen Fueled $100 \mathrm{kN}$ Thrust Capacity Rocket with RPA Program}

\author{
Safa Korkmaz $^{1}$ iD, Hayri Yaman² ${ }^{2}$ \\ ${ }^{1}$ Milli Savunma Bakanlı̆̆l, Posta Kodu ve Ankara, Türkiye \\ ${ }^{2}$ Kırıkkale Üniversitesi Motorlu Araçlar ve Ulaştırma Teknolojisi Bölümü, 71450 ve Kırıkkale, Türkiye
}

Başvuru/Received: 14/08/2021

Kabul / Accepted: 10/10/2021

Çevrimiçi Basım / Published Online: 31/01/2022

Son Versiyon/Final Version: 31/01/2022

\section{$\ddot{O} \mathbf{z}$}

Bu çalışmada, kriyojenik hidrojen yakıtlı $100 \mathrm{kN}$ itki kapasiteli bir roketin teorik itki odası tasarım ve analizleri yapılmıştır. Oksitleyici olarak kriyojenik oksijen kullanılmıştır. Yanma odası basınc1 $20 \mathrm{MPa}$ ve nozul çıkış basıncı $0.101325 \mathrm{MPa}$ sınır şartları olarak belirlenmiştir. Sınır şartları dahilinde RPA bilgisayar programı kullanılarak optimum roket geometrisi oluşturulmuştur. SOLIDWORKS programı ile üç boyutlu roket modeli çizilmiştir. Tasarımı yapılan roketin analizleri RPA ve CEA programları kullanılarak gerçekleştirilmiştir. Roket iç balistik parametreleri olan basınç, sıcaklık ve Mak sayısı değişimleri incelenmiştir. Analiz sonuçlarına göre yanma odası sıcaklığı $3290.9 \mathrm{~K}$ ve nozul çıkış sıcaklığı $1310 \mathrm{~K}$ olarak ölçülmüşsür. RPA ve CEA programlarından elde edilen nozul çıkış basıncı arzu edildiği gibi deniz seviyesi şartlarında $0.1013 \mathrm{MPa}$ olarak sağlanmıştır.

Anahtar Kelimeler

"Itki odası tasarımı, Analiz, RPA, CEA"

\begin{abstract}
In this study was made a conceptual design and analysis of thrust chamber of $100 \mathrm{kN}$ rocket powered by cryogenic hydrogen. Cryogenic oxygen was used as oxidizer. It was determined as boundary conditions which the combustion chamber pressure is 20 $\mathrm{MPa}$ and the nozzle outlet pressure was determined $0.101325 \mathrm{MPa}$. The optimum rocket geometry was created using the RPA computer program within the boundary conditions. Three-dimensional rocket model was drawn with the SOLIDWORKS program. The analysis of the designed rocket was carried out using RPA and CEA programs. Changes of pressure, temperature and Mach number which is rocket internal ballistic parameters were investigated. According to the analysis outputs, the combustion chamber temperature was $3290.9 \mathrm{~K}$ and the nozzle exit temperature $1310 \mathrm{~K}$. The nozzle outlet pressure obtained from the RPA and CEA programs was provided as $0.1013 \mathrm{MPa}$ under sea level conditions as desired.
\end{abstract}

Key Words

"Thrust chamber design, Analysis, RPA, CEA" 


\section{Terminoloji}

\begin{tabular}{|c|c|c|c|}
\hline$F$ & Itki kuvveti & $k$ & Özgül ısı oranı \\
\hline$\dot{m}$ & Kütlesel debi & $P_{c}$ & Yanma odası basincı \\
\hline$A_{t}$ & Nozul boğaz alanı & $P_{a}$ & Ortam basincı \\
\hline$A_{e}$ & Nozul çıkış alanı & $P_{e}$ & Nozul çıkış basıncı \\
\hline$A_{c}$ & Yanma odası alanı & $t_{s}$ & Yanma süresi \\
\hline$L^{*}$ & Yanma odası karakteristik uzunluğu & $V_{e}$ & Egzoz hizl \\
\hline$L_{c}$ & Yanma odası uzunluğu & $V_{c}$ & Yanma odası hacmi \\
\hline$L_{n}$ & Nozul uzunluğu & $g$ & Yerçekimi ivmesi \\
\hline$L_{c y l}$ & Yanma odası silindirik bölüm uzunluğu & $c^{*}$ & Karakteristik egzoz hızl \\
\hline$R$ & Gaz sabiti & $C_{F}$ & Itki katsayısı \\
\hline$M_{g}$ & Moleküler ağırlık & $c$ & Efektif egzoz hızı \\
\hline$\lambda$ & Düzeltme faktörü & $T_{c}$ & Yanma odast sicaklı $\breve{g} l$ \\
\hline$\varepsilon$ & Nozul genişleme oranı & $\alpha$ & Nozul genişleme açısı \\
\hline$I_{s p}$ & Özgül itki & $b$ & Nozul daralma açısı \\
\hline
\end{tabular}

\section{Giriş}

Roketler, Newton'un üçüncü hareket yasası olan her etkiye eşit ve zıt bir tepki prensibine göre çalışan motorlara verilen isim olarak adlandırılmaktadır (Yaman, 2013). Roketler çalışmaları esnasında hava ortamına ihtiyaç duymayan sistemlerdir ve bu özellikleri sayesinde atmosfer dışı her ortamda çalışabilmektedirler (Ward, 2010). Roketler bir yükü hedefe ulaştırmak için kullanılan araçlardır. Bu yükler askeri amaçlı silah başlığı, uzay çalışmaları kabini, ticari ve haberleşme uydular olabilmektedir. Son yıllarda roketler ile insan taşımacılığı yapmak için çalışmalar devam etmektedir (Gill ve Nurick, 1976; Samur, 2015; Sutton ve Biblarz, 2016).

Roketlerde itki, temel olarak yanma odasında üretilen yüksek sıcaklık ve basınçtaki gazın nozul aracılıyla dışarı atılması esnasında oluşan momentumdan elde edilir (Gill ve Nurick, 1976). Roketler enerji kaynağının çeşidine göre (kimyasal, nükleer, elektrik vb), temel işlevlerine (yükseltici, yörünge istasyonu tutucu), araç tipine (uçak, füze, uzay aracı), yakıt tipine ve kademe sayısına göre sınıflandırılmaktadırlar(Sutton ve Biblarz, 2016). İçlerinde en yaygın kullanılan sınıflandırma yöntemi, roketin kullandığı enerji kaynağının çeşidine göre yapılan sınıflamalardır (Dönmez, 2018; Karayel, 2018).

Sıvı yakıtlı roket motorları; oksitleyiciyi ve yakıtı sıvı formda kullanan kimyasal roket motorlarıdır. Sıvı yakıtlı roketler, diğer kimyasal roketlerde olduğu gibi kimyasal enerjinin kinetik enerjiye dönüştürülmesi prensibi ile çalışmaktadırlar (Cengiz, 2010). Genel olarak katı yakıtlı roket motorlarından daha fazla itki üretmektedirler. Fakat sıvı yakıtlı roket motorları karmaşık alt sistemlere sahiptir. Yakıt ve oksitleyicinin yanma odasına taşındığı besleme ve pompa sistemleri, yanma odası ve nozulun soğutulması için kullanılan soğutma sistemi, kontrol ve güvenlik için kullanılan valfler gibi karmaşı yapıları barındırmaktadır. Bu sebeplerden dolayı sıvı yakıtlı roketler katı yakıtlı roketlere göre çok karmaşıktır ve maliyetleri oldukça yüksektir (Santos vd, 2011; Türk, 2016).

Strunz (1998) sıvı yakıtlı roketin kararsızlıklarını teorik olarak incelemiş ve tanımlamıştır. Akış olayındaki geçici durumları tahmin etmek için numerik yöntemler kullanmıştır. Basitleştirilmiş yanma modeli için analizlerini Karakteristik Metot ve tek boyutlu termokimyasal kodlarını kullanarak yapmıştır (Strunz, 1998).

Guabio Cai ve arkadaşları (2007) sıvı yakıtlı roket nozul optimizasyonuna bağlı performans tahminini araştırmışlardır. Hesaplamalı akışkanlar dinamiği (CFD) metotlarını nozul performansını daha iyi tahmin edebilmek için optimizasyon süreci ile eşleştirilmiştir. CFD tabanlı optimizasyon prosedürü sayesinde daha iyi nozul performansı elde edilmiştir. Nozul performansı \%1.5 artmıştır (Cai vd, 2007).

Youngblood (2015) yapmış olduğu çalışmada küçük ölçekli etanol ve nitro oksit $\left(\mathrm{N}_{2} \mathrm{O}\right)$ ile çalışan sıvı yakıtlı roket tasarlamıştır. Tasarladığı roketi Nasa Chemical Equilibrium with Applications (CEA) ve Cantera referanslı Matlab ortamında model oluşturarak analiz etmiş̧tir. Daha sonra roketi üreterek ve statik testlerini yapmışıtır. Test verileri ile programların sonuçlarını karşılaştırıp Cantera programının Nasa CEA programı kadar iyi olduğunu ve iyi bir rakip olacağını göstermiştir (Youngblood, 2015).

Porto Riko Mayagüez üniversitesinden roket takımı Loxodon-1 adı verdikleri, sıvı oksijen (LOX) ve Jet-A yakıtı ile çalışan $2.2 \mathrm{kN}$ itki üreten sıvı yakıtlı roketi Rocket Propulsion Analysis (RPA) programı kullanarak geliş̧irmişlerdir. Roketin çalışma basıncı $3.82 \mathrm{MPa}$ ve yanma odası $2218 \mathrm{~K}$ sıcaklığa ulaşmıştır. Tasarımı biten roket üretim aşamasına geçmiş olup ateşleme testinin 2021 ilkbaharda yapılması planlanmışlardır (Rodríguez Otero vd, 2020). 
Ojeda ve arkadaşları 2017 yılında akademik bir tasarım ortamında düşük itki gücüne sahip bipropellant bir sıvı roket motorunu tasarlamışlar, üretmişler ve test etmişlerdir. Yakıt olarak etanol oksitleyici olarak hidrojen peroksit kullanmışlardır. Florida Üniversitesi sıvı tahrik tasarım takımında bulunan Ojeda ve arkadaşları tasarım aşamasında roket geometrisinin boyutunu ve performansını öngörmek için RPA programını kullanmışlardır (Ojeda vd, 2017).

Eric P. 2018 yılında Berkley Üniversitesinde $\mathrm{N}_{2} \mathrm{O}$ ve Jet-A ile çalışan $11 \mathrm{kN}$ itki kapasiteli sıvı yakıtlı sonda roket motorunun tasarımını yapmıştır. Roket, deniz seviyesinden $100 \mathrm{~km}$ yüksekteki yörüngeye ulaşmak için tasarlanmıştır. Roket motor geometrisinin parametrelerini belirlemek ve roket motor performansını test etmek için RPA programını kullanmıştır (Pillai, 2018).

Ngwu ve arkadaşları 2020 yılında $100 \mathrm{~N}$ itki kuvvetine sahip Hidrojen Peroksit ve Kerosen ile çalışan sıvı yakıtlı roket motorunun tasarımını nümerik olarak yapmışlardır. Yaptıkları çalışmayı doğrulamak için RPA programını kullanmışlardır (Ngwu vd, 2020).

Baxi ve arkadaşları 2021 yılında yapmış oldukları çalışmada Vikas 4B sıvı yakıtlı roket motorunu RPA programını kullanarak modellemişlerdir. Yakıt çifti olarak 1.71 karışım oranında Oksijen ve Hidrojen kullanmışlardır. Daha sonra RPA programını kullanarak en verimli roket geometrisini belirlemişlerdir. Roket geometrisi ile SolidWorks programı aracılı̆̆ ile katı modelini oluş̧urmuşlardır. Roket motor duvar malzemesi olarak 152.4 mm (6 inç) kalınlığında 1 ssıl işlem görmüşs karbon fiber kullanmışlardır. ANSYS yazılımını kullanarak roket duvarlarında oluşan gerilmeleri, gaz akış hızını ve roket iç basınç dağılımını analiz etmişlerdir. Elde edilen verilerle roket motorunun güvenli olduğu sonucuna varmışlardır (Baxi vd, 2021)

Bu çalışmada $100 \mathrm{kN}$ itki kapasiteli sıvı yakıtlı roket motor tasarımı hedeflenmiştir. Yapılan çalışmada elde edilen çıktılar; bulgular, tartışma ve sonuçlar kısmında ayrıntılı olarak verilmiştir.

\section{Materyal ve Metot}

Yanma odası basıncı $20 \mathrm{MPa}$ olan ve yakıt çifti olarak belirlenen sıvı oksijen- sıvı hidrojen ile 100 kN'luk itkiye sahip bir sıvı yakıtlı roket motor tasarımı yapılmıştır. Öncelikli olarak analitik hesaplamalar yapılmış ve daha sonra RPA programı yardımıyla roket boyutları optimize edilmiştir. Sıvı yakıtlı roketin yanma odası ile nozul tasarımı yapıldıktan sonra analizler için paket programlar kullanılmıștır.

Kullanılan programlar; Alexander Ponomarenko tarafından geliştirilen RPA programı, roket motorlarının performans tahmini ve optimizasyonu için kullanılan bilgisayar destekli bir programdır. Roket motorlarının tasarımı ve analizi az sayıda parametre girilerek yapılabilmektedir. Bu sayede hem zaman hem de maliyet açısından üstün ve doğru sonuçların elde edilmesi sağlamaktadır (Ponomarenko, 2009).

Tasarımı yapılan roket motorunun analizleri Nasa'nın bilgisayar programı olan CEA ve RPA programları ile yapılmış ve karşılaştırılmıştır. CEA kimyasal denge kompozisyonlarını ve karmaşık karışımların özelliklerini hesaplamaktadır. CEA, son 45 yıl içinde NASA Lewis (şimdi Glenn) Araştırma Merkezi'nde geliştirilen bir dizi bilgisayar programında en sonuncusunu temsil etmektedir.

Tasarımı yapılan roketin yanma odası basıncı 20MPa, çevre basıncı $101325 \mathrm{~Pa}$ olarak belirlenmiştir. Sıvı hidrojen yakıtının sıcaklığı $45 \mathrm{~K}$, oksitleyici sıvı oksijen sıcaklığı ise $90 \mathrm{~K}$ 'dir. Hidrojen ve oksijen yakıt çiftinin stokiyometrik oranı 7.937 olmasına rağmen, Şekil 1 'de görüldüğü gibi en yüksek özgül itki değeri oksitleyici yakıt oranı 4.8 olduğunda elde edilmektedir. Bu amaçla roket yakıt karışım oranı 4.8 olarak belirlenmiştir.

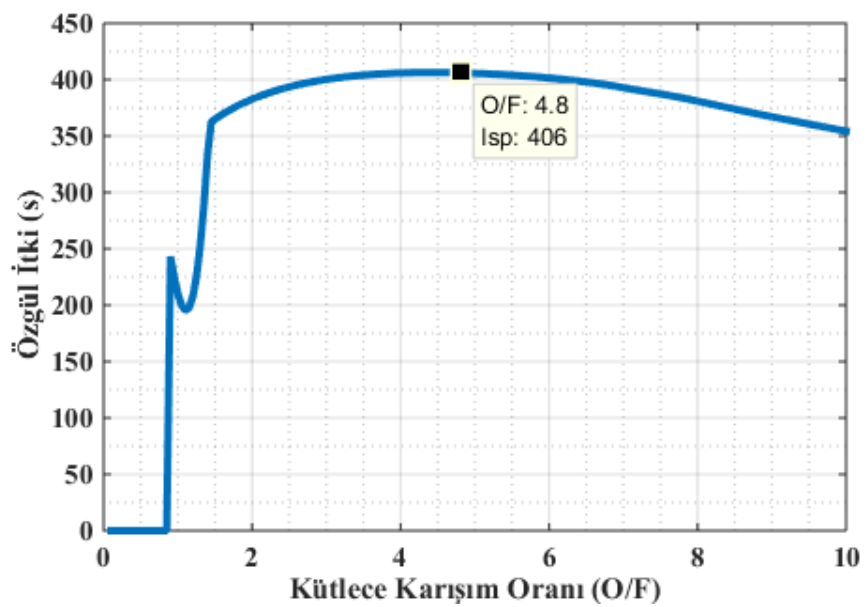

(a)

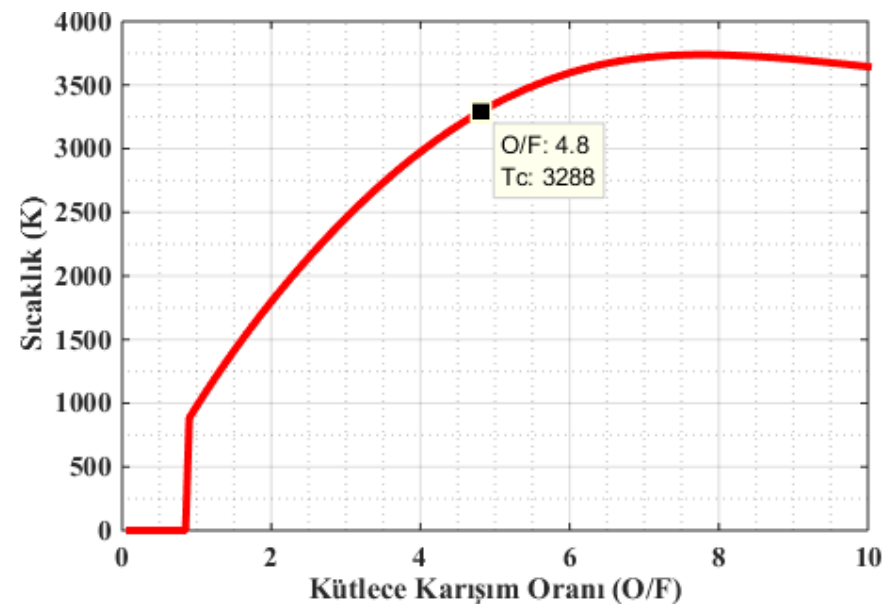

(b)

Şekil 1 Sıvı Hidrojen - Sıvı Oksijen kütlece karışım oranlarına göre (a) özgül itki ve (b) sıcaklık değişim grafiği 
Roket motorundan 100 kN itki elde edebilmek için (Raposo, 2016),

$$
\begin{aligned}
\dot{\mathrm{m}}_{\text {toplam }} & =\frac{\mathrm{F}}{\mathrm{gI}_{\mathrm{sp}}} \\
\dot{\mathrm{m}}_{\text {toplam }} & =\frac{100 \mathrm{kN}}{9.81 \times 406}=25.11 \mathrm{~kg} / \mathrm{s}
\end{aligned}
$$

Denklem 1 ve 2 ‘de görüldüğü gibi yakıtlarımızın kütlesel debisi $25.11 \mathrm{~kg} / \mathrm{s}$ olması gerekmektedir. Karışım oranı 4.8 olduğundan

$$
\begin{aligned}
& \dot{\mathrm{m}}_{\mathrm{f}}=\frac{\dot{\mathrm{m}}_{\text {toplam }}}{\mathrm{r}_{\text {karısım }}+1}+1 \\
& \dot{\mathrm{m}}_{\mathrm{f}}=\frac{25.11}{5.8}=4.33 \mathrm{~kg} / \mathrm{s} \\
& \dot{\mathrm{m}}_{\mathrm{o}}=\dot{\mathrm{m}}_{\mathrm{f}} \times \mathrm{r}_{\begin{array}{c}
\text { karısım } \\
\text { oramı }
\end{array}}=4.33 \times 4.8=20.78 \mathrm{~kg} / \mathrm{s}
\end{aligned}
$$

İtki odası tasarımı için yanma odası şekli silindirik ve nozul tipi olarak da De laval \%80'lik parabolik yapı seçilmiştir. Roket nozul boğaz çapı Denklem 6 ile belirlenmektedir (Hetem vd, 2011).

$$
\mathrm{A}_{\mathrm{t}}=\frac{\mathrm{F}}{\mathrm{P}_{\mathrm{c}} \mathrm{C}_{\mathrm{F}}}
$$

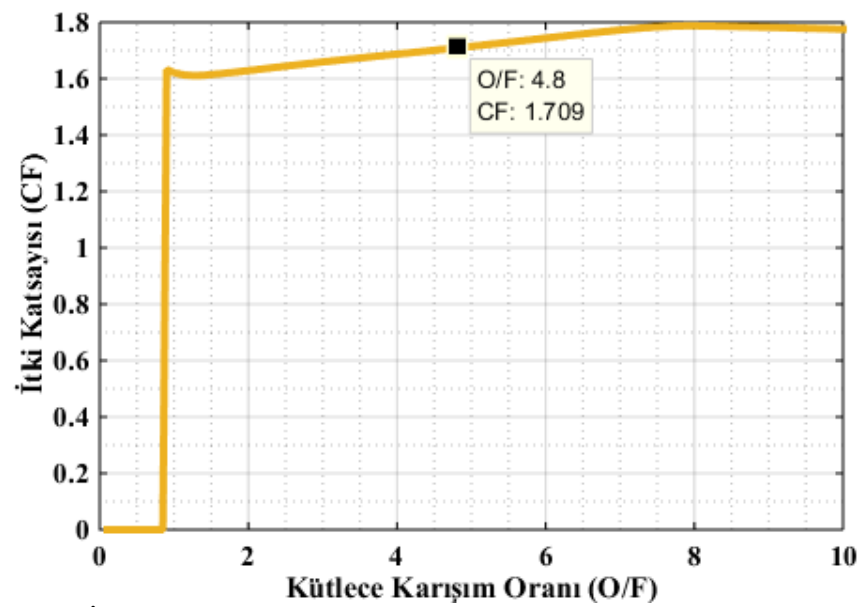

Şekil 2 İtki Katsayısının yakıt çiftinin karışım oranına göre değişimi

İtki katsayısının değeri Şekil 2'deki grafikten de anlaşılacağı üzere karışım oranına göre değişmektedir. İtki katsayısı stokiyometrik karışım oranına kadar artmakta ve bu orandan sonra azalmaktadır.

$$
\begin{aligned}
& \mathrm{A}_{\mathrm{t}}=\frac{100 \mathrm{kN}}{20 \mathrm{MPa} \times 1.709}=2926 \mathrm{~mm}^{2} \\
& \mathrm{D}_{\mathrm{t}}=\sqrt{\frac{4 \mathrm{~A}_{\mathrm{t}}}{\pi}}=61.04 \mathrm{~mm}
\end{aligned}
$$

Roket genişleme oranı nozul çıkış alanının nozul boğaz alanına oranı olarak ifade edilmektedir ve $\epsilon$ ile gösterilmektedir.

$$
\epsilon=\frac{A_{e}}{A_{t}}=\frac{\left[\frac{2}{k+1}\right]^{\frac{1}{k-1}}\left[\frac{P_{c}}{P_{e}}\right]^{\frac{1}{k}}}{\sqrt{\frac{k+1}{k-1}\left[1-\left[\frac{\mathrm{P}_{\mathrm{e}}}{\mathrm{P}_{\mathrm{c}}}\right]^{\frac{\mathrm{k}-1}{\mathrm{k}}}\right]}}
$$

Roketler nozul çıkış basıncı atmosfer basıncına eşit olduğunda en iyi performans elde edilmektedir. Roket ideal şartlarda deniz seviyesinde çıkış basıncının $\mathrm{Pe}=101235 \mathrm{~Pa}$ olması istenmektedir. Roket tasarımında özgül 1sı oranı genellikle $\mathrm{k}=1.2$ olarak alınmaktadır. Bu şartlar altında Denklem 9 çözüldüğünde $\epsilon=20.02$ olarak bulunmaktadır. 


$$
\begin{aligned}
& A_{e}=\epsilon A_{t} \\
& A_{e}=58578 m^{2} \\
& D_{e}=273 \mathrm{~mm}
\end{aligned}
$$

Roket nozul çıkış kesit alanı $58575 \mathrm{~mm}^{2}$ ve nozul çıkış çapı $273 \mathrm{~mm}$ olarak bulunmuştur. Roketin herhangi bir noktasındaki alan Denklem 13 ile ifade edilmektedir.

$$
\frac{A_{x}}{A_{t}}=\frac{\left[\frac{2}{k+1}\left(\frac{P_{c}}{P_{x}}\right)^{\frac{k+1}{2 k-2}}\right]}{\sqrt{\frac{2}{k-1}\left[\left(\frac{P_{c}}{P_{x}}\right)^{\frac{k-1}{k}}-1\right]}}
$$

Denklem 13 ile yanma odası alanı Ac $=4.6 \mathrm{At}=13460 \mathrm{~mm}^{2}$ olarak hesaplanmıştır. Yanma odası çapı Dc $=131$ mm'dir. Teorik yanma odası hacmi; yakıtların kütlesel debisi, ortalama özgül hacim ve verimli bir yanma için gerekli sürenin bir fonksiyonu olarak Denklem 14'te tanımlanmaktadır.

$$
\mathrm{V}_{\mathrm{c}}=\dot{\mathrm{m}} \vartheta \mathrm{t}_{\mathrm{s}}
$$

Denklem 14 'te $V_{c}$ yanma odası hacmi, m yakıtların toplam kütlesel debisi, $\vartheta$ ortalama özgül hacmi ve $t_{s}$ ise yakıtın yanma odasında kalma süresi olarak ifade edilmektedir. Karakteristik uzunluk olarak tanımlanan $L^{*}$ yakıt kalma süresinin belirlemede kullanılabilir. $L^{*}$ yanma odasının hacminin nozul boğaz alanına oranı olarak Denklem 15'de tanımlanmaktadır.

$$
\mathrm{V}_{\mathrm{c}}=\mathrm{A}_{\mathrm{t}} \mathrm{L}^{*}
$$

L* deneysel çalışmalarla elde edilmiştir ve yakıt kombinasyonlarına göre değeri değişmektedir. Sıvı Oksijen - Sıvı Hidrojen için yanma odası karakteristik uzunluk Tablo 1'den elde edilmiştir.

Tablo 1 Çeşit yakıt çiftleri için yanma odası karakteristik uzunluğu (Huang ve Huzel, 1971)

\begin{tabular}{lc}
\hline Yakıt Çifti & Yanma Odası Karakteristik Uzunluğu $\left(L^{*}\right)$ \\
\hline Sıvı Flor - Hidrazin & $610-710 \mathrm{~mm}$ \\
\hline Sıvı Flor - Sıvı Hidrojen & $560-750 \mathrm{~mm}$ \\
\hline Hidrojenperoksit - RP-1 & $1520-1750 \mathrm{~mm}$ \\
\hline Nitrik Asit - Hidrazin & $750-900 \mathrm{~mm}$ \\
\hline Sıvı Oksijen - Sıvı Hidrojen & $550-1020 \mathrm{~mm}$ \\
\hline Sıvı Oksijen - RP-1 & $1000-1250 \mathrm{~mm}$ \\
\hline
\end{tabular}

$$
\mathrm{V}_{\mathrm{c}}=2926 \times 1020=2984520 \mathrm{~mm}^{3}
$$

Tasarımı yapılan roketin gerekli yanma odası hacmi Denklem 16'da görülmektedir. Yanma odasını şekli silindirik olarak seçilmiştir. Şekil 3'te görülen roket geometrisinden yanma odası uzunluğu ve nozul uzunluğu hesaplanabilmektedir. Şekil 3'te görülen Lcyl yanma odası silindirik kısmının uzunluğunu, Lc yanma odasının toplam uzunluğunu ifade etmektedir. Nozul daralma yarım açısı b $12^{\circ}$ ve nozul genişleyen yarım açısı $\mathrm{Tn} 28.83^{\circ}$ olarak alınmıştır. 


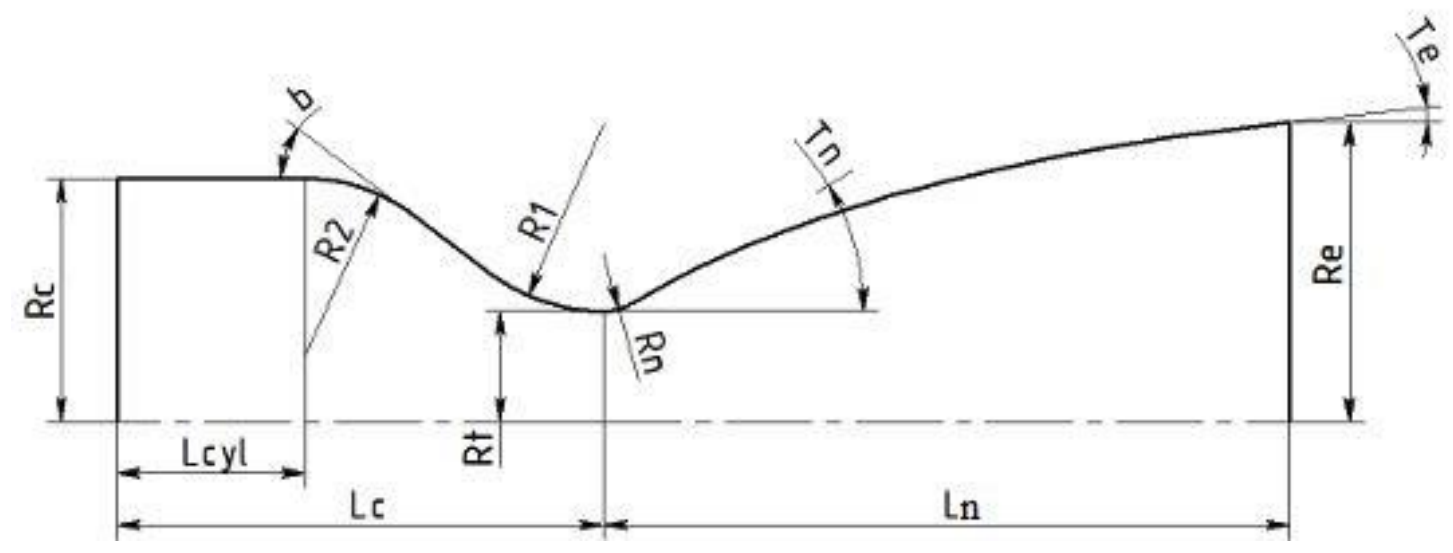

Şekil 3 Roket geometrisi

Şekil 3'te görülen radyuslar sırasıyla R1 $=124.19 \mathrm{~mm}, \mathrm{R} 2=751.14 \mathrm{~mm}$ ve $\mathrm{Rn}=11.86 \mathrm{~mm}$ olacak şekilde alınmıştır.

$$
\mathrm{L}_{\mathrm{c}}=\mathrm{L}_{\mathrm{cyl}}+\mathrm{R}_{2} \tan \left(\frac{\mathrm{b}}{2}\right)+\left(\frac{\mathrm{R}_{\mathrm{c}}-\mathrm{R}_{\mathrm{t}}}{\tan \mathrm{b}}\right)+\mathrm{R}_{1} \tan \left(\frac{\mathrm{b}}{2}\right)
$$

Lcyl hesaplamak için Denklem 18 kullanılmaktadır. $\mathrm{V}_{2}$ nozulun daralan kısmından nozul boğazına olan kısmın hacmini ifade etmekte ve eğrinin integrali alınarak hesaplanmaktadır. $\mathrm{V}_{2}$ hacmi $2278667 \mathrm{~mm} 3$ olmaktadır.

$$
\mathrm{V}_{\mathrm{c}}=\mathrm{A}_{\mathrm{c}} \mathrm{L}_{\mathrm{cyl}}+\mathrm{V}_{2}
$$

Denklem 18'den Lcyl 52.4 mm olarak hesaplanmaktadır. Denklem 17'den Lc değeri 309 mm olarak bulunmaktadır.

$$
\begin{aligned}
& \mathrm{L}_{\mathrm{n}}=\frac{0.8(\sqrt{\epsilon}-1) \mathrm{R}_{\mathrm{t}}+\mathrm{R}_{\mathrm{n}}}{\tan \left(\frac{\mathrm{T}_{\mathrm{n}}}{2}\right)} \times \lambda \\
& \lambda=\frac{1}{2}\left(1+\cos \left(\frac{\mathrm{T}_{\mathrm{n}}}{2}\right)\right)
\end{aligned}
$$

Nozulda meydana gelen eksenel olmayan gaz akışlarını da hesaba katmak için $\lambda$ kullanılmaktadır ve düzeltme faktörü olarak bilinmektedir. Roket nozul uzunluğu Denklem 19 ve Denklem 20 yardımıyla, 370.4 mm olarak bulunmaktadır.

RPA programına giriş parametreleri, yanma odası basıncı $20 \mathrm{MPa}$, yakıt çifti Sıvı Hidrojen - Sıvı Oksijen, oksitleyici yakıt oranı 4.8, nozul çıkış basınc1 $1 \mathrm{~atm}, \mathrm{~A}_{\mathrm{c}} / \mathrm{A}_{\mathrm{t}}$ oranı 4.6 ve nominal itki $100 \mathrm{kN}$ olarak girilmiştir. Şekil 4 'te RPA programı kullanıcı arayüzü gösterilmektedir. Programa girilen gerekli bilgiler sarı kutucuk ile işaretlenmiştir. 
Q8 Rocket Propulsion Analysis (Standard Edition) - 100 k.cfg

File View Run Help

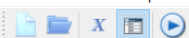

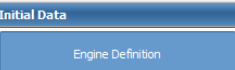

Engine Definition

Propellant Specification

Engine name:
Description:

Nozzle Flow Model

Description:

$100 \mathrm{k}$

\begin{tabular}{lll}
\hline & \\
Chamber pressure: & 20 & MPa \\
DDetermine thrust chamber size matching the specifed requirements
\end{tabular}

\begin{tabular}{l|l|l|l|l|}
\hline O Nominal thrust: & 100 & $\mathrm{kN}$ & at ambient pressure: 1 & 1
\end{tabular}

\begin{tabular}{l|ll}
\hline & Mass flow rate: & $\mathrm{kg} / \mathrm{s} \quad \mathrm{m}$ (m-dot, total at $100 \%$ throtte) \\
\hline
\end{tabular}

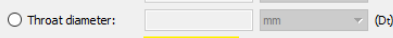

Number of chambers: 1

$\square$ Perform chamber thermal analysis $\quad$ Parameters (f not specifed, radiation cooling is assumed for whole chamber)

$\square$ Determine parameters of propellant feed system

$\begin{array}{ll}\text { Gas-pressurized: } & \text { by stored gas } \\ \text { Turbopump: } & \text { gas generator cyde }\end{array}$

System spec (f not specifed, default parameters are assumed)

$\square$ Estimate engine dry weight

Performance Analysis

Engine Design

Tools

Rocket Propulsion Analysis (Standard Edition) - $100 \mathrm{k}$. fog

File View Run Help

1 -

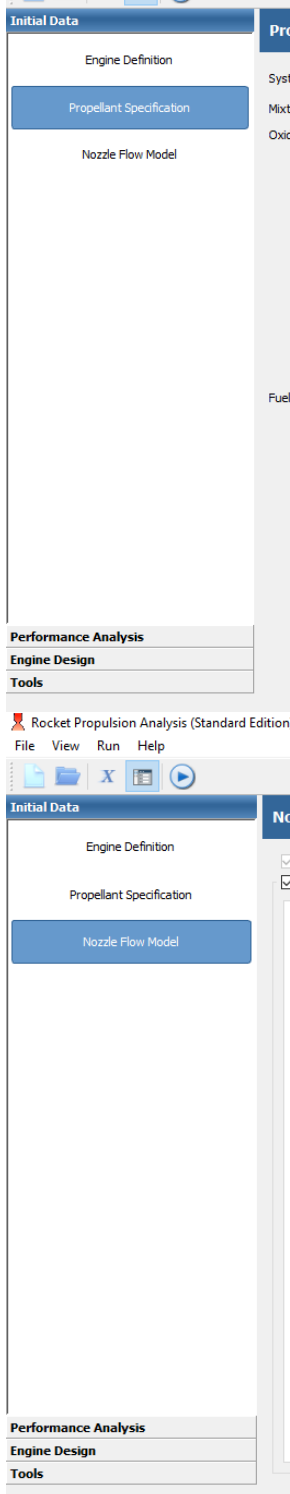

Propellant Specification

System:

Mixture ratio:

Oxidizer:
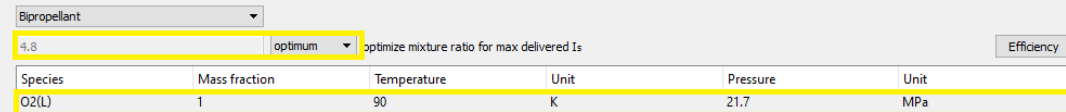

21.7

\begin{tabular}{|c|c|c|c|c|c|c|}
\hline \multicolumn{7}{|c|}{ Sum of all the mass fractions: 1} \\
\hline Add & Define new & Remove & Normalize & & & \\
\hline Species & & Mass fraction & Temperature & Unit & Pressure & Unit \\
\hline $\mathrm{H} 2(\mathrm{~L})$ & & 1 & 45 & K & 23.75 & $\mathrm{MPa}$ \\
\hline
\end{tabular}

Sum of all the mass fractions: 1

\begin{tabular}{|l|l|l|}
\hline Add & Define new & Remove \\
\hline
\end{tabular}

Nozzle Flow Model Specification

Calculate combustion parameters

Calculate nozzle flow

Nozzle conditions Nozzle Shape and Efficiencies Ambient condition / Throttle settings

$\square$ Nozzle inlet condition

mass flux:

(-) contraction area ratio

$\mathrm{kg} /\left(\mathrm{m}^{2} \mathrm{~s}\right)$

\begin{tabular}{l|l}
4.6 & $\left(\mathrm{Ac} / \mathrm{At}_{\mathrm{t}}\right)$
\end{tabular}

If nether

Nozzle exit condition

pressure:

expansion area ratio:

$O$ expansion pressure ratio

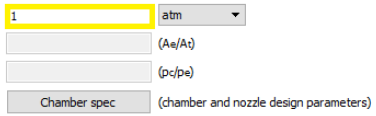

$\square$ Frozen equilibrium flow

freezing at the pressure ratio:

Oremang at

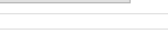

(ptpit)

If not specifed, the shifing equilbrium model is applied for the whole nozzle.

Nozzle stations

Standard nozzle stations are designated as follows: $c=$ combustion chamber, $t=$ nozzle throat, $e=$ nozzle exit, $f t=$ frozen.

\begin{tabular}{l|c|} 
Specify additional stations: & Stations \\
\end{tabular}

Şekil 4 RPA programı kullanıcı arayüzü 
Şekil 4'te verilen parametreler için RPA programı aracıllı̆̆ ile oluşturulan roket motor geometrisi Tablo 2'de sunulmuştur.

Tablo 2 RPA programı itki odası geometrisinin değerleri

\begin{tabular}{clcl}
\hline İtki Odası Geometri Parametresi & Değer & İtki Odası Geometri Parametresi & Değer \\
\hline $\mathrm{Dc}$ & $133.18 \mathrm{~mm}$ & $\mathrm{Ae} / \mathrm{At}$ & 18.61 \\
$\mathrm{Dt}$ & $62.10 \mathrm{~mm}$ & $\mathrm{R} 1$ & $124.19 \mathrm{~mm}$ \\
$\mathrm{De}$ & $267.90 \mathrm{~mm}$ & $\mathrm{R} 2$ & $751.14 \mathrm{~mm}$ \\
$\mathrm{~L}^{*}$ & $1018.26 \mathrm{~mm}$ & $\mathrm{Rn}$ & $11.86 \mathrm{~mm}$ \\
$\mathrm{Ln}$ & $353.44 \mathrm{~mm}$ & $\mathrm{~b}$ & $12.00^{\circ}$ \\
$\mathrm{Lc}$ & $311.06 \mathrm{~mm}$ & $\mathrm{Tn}$ & $28.83^{\circ}$ \\
Lcyl & $51.84 \mathrm{~mm}$ & $\mathrm{Te}$ & $8.00^{\circ}$ \\
\hline
\end{tabular}

Tablo 2'de RPA programının 100 kN'luk roket itki odası geometrisinin değerleri görülmektedir. Şekil 5'te Tablo 2'de sunulan roket motorunun Solidworks programında oluşturulan üç boyutlu katı modelin kesit görünümü verilmiştir.

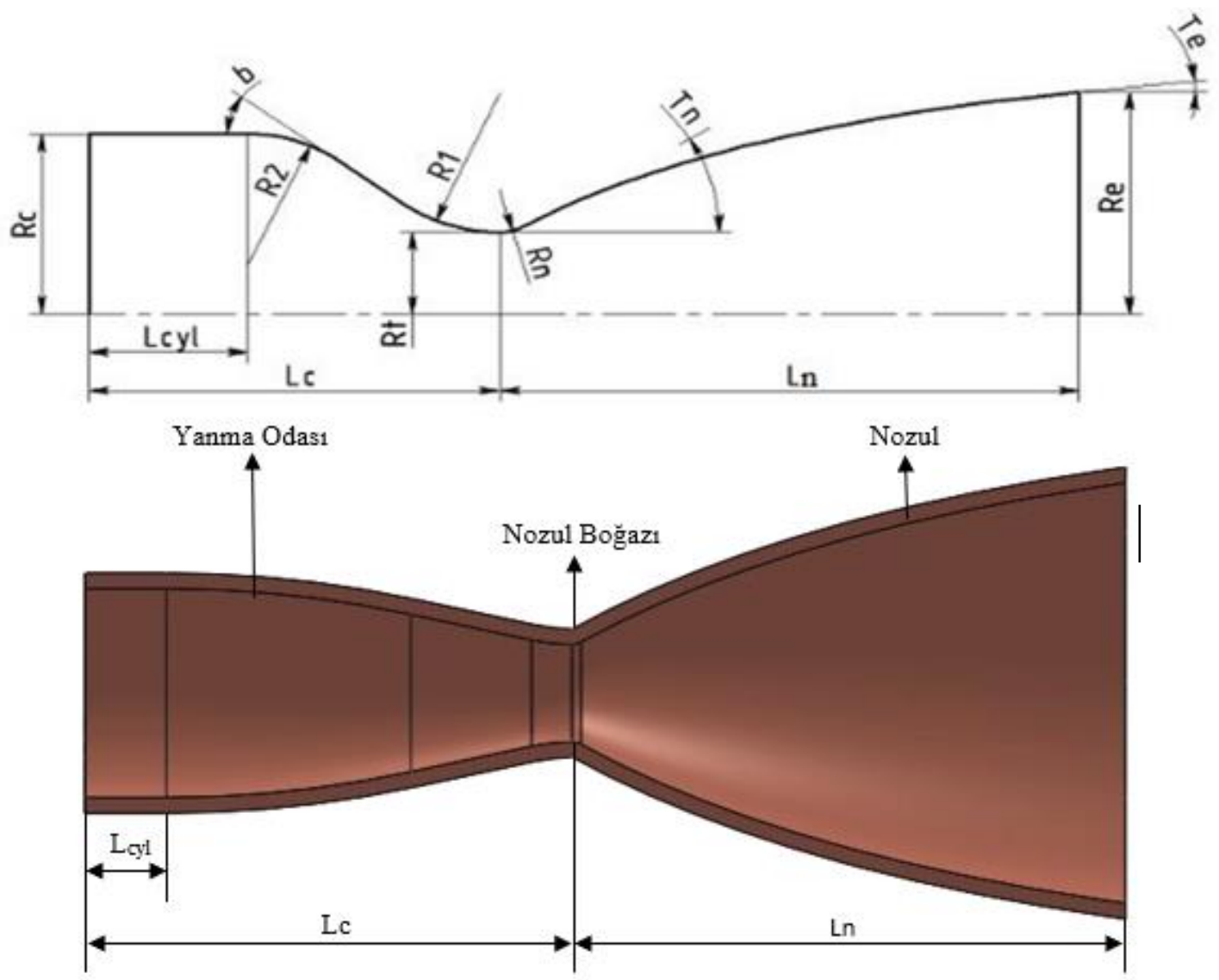

Şekil 5100 kN'luk roket itki odası kesit görünümü

\section{Bulgular}

Tasarım geometrisi belirlenen roketin sınır şartları öncelikli olarak RPA programına girilmiştir. Çıktı olarak programdan atmosfer ortamı (deniz seviyesinde) ve atmosfer dışı (uzay vakum ortamında) roket çalışma değerleri elde edilmiştir. Tablo 3-4'de sırasıyla performans ve analiz sonuçları olarak verilmiştir. Performans sonuçlarına göre sıvı yakıtlı roket motoru deniz seviyesinde 99.99360 kN itki kuvveti üretirken, vakum ortamında $105.71135 \mathrm{kN}$ itki kuvveti üretmektedir. Oksitleyici yakıt oranı 4.8 iken ideal kütlesel debi $25.11385 \mathrm{~kg} / \mathrm{s}$ 'dir. 
Tablo 2 RPA vasıtasıyla elde edilen performans sonuçları.

\begin{tabular}{ll}
\hline İtki Kuvveti ve Kütlesel Debi & Değer \\
\hline Vakum Altında İtki & $105.71135 \mathrm{kN}$ \\
\hline Vakum Altında Özgül İtki & $429.22756 \mathrm{~s}$ \\
\hline Deniz Seviyesinde İtki & $99.99360 \mathrm{kN}$ \\
\hline Deniz Seviyesinde Özgül İtki & $406.01136 \mathrm{~s}$ \\
\hline Toplam Kütlesel Debi & $25.11385 \mathrm{~kg} / \mathrm{s}$ \\
\hline Oksitleyicinin Kütlesel Debisi & $20.78835 \mathrm{~kg} / \mathrm{s}$ \\
\hline Yakıtın Kütlesel Debisi & $4.32551 \mathrm{~kg} / \mathrm{s}$ \\
\hline
\end{tabular}

Tablo 4'te verilen analiz sonucuna göre nozul çıkış basıncı 0.1013 MPa olarak elde edilmiştir. Egzoz çıkış basıncı ile ortam basıncının eşit olması $\left(\mathrm{P}_{\mathrm{e}}=\mathrm{P}_{\mathrm{a}}\right)$ nedeniyle optimum itki koşulunun sağlandığı görülmüştür. Nozul çıkışı gaz hızı süpersonik (1-5 Mak aralığı) kategorisinde içerisinde yer alan 3.76 Mak değeri olarak tespit edilmiştir.

Tablo 4100 kN'luk roketin deniz seviyesi için RPA analiz programı ile elde edilen sonuçlar.

\begin{tabular}{cccccc}
\hline & Yanma Odası & Nozul Girişi & Nozul Boğazı & Nozul Çıkışı & Birim \\
\hline Basınç & 20 & 19.6099 & 11.26 & 0.1013 & $\mathrm{MPa}$ \\
Sıcaklık & 3289.8884 & 3284.9488 & 3039.847 & 1309.6263 & $\mathrm{~K}$ \\
Özgül Isı (P=Sabit) & 5.9598 & 5.9562 & 5.4058 & 3.3981 & $\mathrm{~kJ} /(\mathrm{kg} \cdot \mathrm{K})$ \\
Özgül Isı (V=Sabit) & 5.0518 & 5.0488 & 4.5675 & 2.6877 & $\mathrm{~kJ} /(\mathrm{kg} \cdot \mathrm{K})$ \\
Özgül Isı Oranı $(\mathrm{K})$ & 1.1797 & 1.1797 & 1.1835 & 1.2643 & \\
Yoğunluk & 8.4429 & 8.2911 & 5.1707 & 0.1089 & $\mathrm{~kg} / \mathrm{m}^{3}$ \\
Ses Hızı & 1665.9959 & 1664.7144 & 1602.0169 & 1084.5442 & $\mathrm{~m} / \mathrm{s}$ \\
Gaz Hızı & 0 & 216.9051 & 1602.0169 & 4086.2915 & $\mathrm{~m} / \mathrm{s}$ \\
Mak Sayısı & 0 & 0.1303 & 1 & 3.7678 & \\
Alan Oranı $\left(\frac{A_{c}}{A_{t}}, \frac{A_{t}}{A_{t}}, \frac{A_{e}}{A_{t}}\right)$ & 4.6 & 4.6 & 1 & 18.6127 & \\
\hline
\end{tabular}

Tasarımı yapılan roket CEA programı ile Tablo 5’te görüldüğü gibi aynı sınır şartları kullanılarak analizleri gerçekleştirilmiş̧tir. CEA programı ile yapılan analiz sonuçları RPA programında benzer şekilde çok yakın değerler elde edilmiştir.

Tablo 5100 kN'luk roketin CEA analiz programı ile elde edilen analiz sonuçları.

\begin{tabular}{lccccl}
\hline & Yanma Odası & Nozul Girişi & Nozul Boğazı & Nozul Çıkışı & Birim \\
\hline Basınç & 20 & 19.609 & 11.26 & 0.10138 & $\mathrm{MPa}$ \\
Sicaklık & 3290.9 & 3285.94 & 3040.94 & 1310.7 & $\mathrm{~K}$ \\
Yoğunluk & 8.44430 & 8.29210 & 5.17140 & 0.10894 & $\mathrm{~kg} / \mathrm{m}^{3}$ \\
Özgül Isı & 5.9624 & 5.9588 & 5.408 & 3.3977 & $\mathrm{~kJ} /(\mathrm{kg} \cdot \mathrm{K})$ \\
(P=Sabit) & & & & & \\
Özgül Isı Oranı & 1.1716 & 1.1716 & 1.1784 & 1.2642 & \\
(K) & & & & & \\
Ses Hızı & 1665.8 & 1664.5 & 1601.9 & 1084.7 & $\mathrm{~m} / \mathrm{s}$ \\
Mak Sayıs1 & 0 & 0.13 & 1 & 3.767 & \\
Ittki Katsayıs1 & 0.0908 & 0.0908 & 0.67 & 1.7091 & \\
(Cf) & 0.595 & 0.5394 & 0.5561 & 0.6473 & \\
Pr & $9.75 \times 10^{-5}$ & $9.75 \times 10^{-5}$ & $9.75 \times 10^{-5}$ & $9.75 \times 10^{-5}$ & $\mathrm{~kg} /(\mathrm{m} \mathrm{s})$ \\
Viskozite & & & & & \\
\hline
\end{tabular}


Tablo 6'da RPA programı ile CEA programı analiz sonuçlarının karşılaştırılması verilmiştir. Her iki program tarafından da roket motor basınç dağılım ve Mak sayısı değelerinin aynı olduğu görülmüştür. Ancak roket motor iç sıcaklık dağılımı sonuçları incelendiğinde, CEA programı sonuçlarının RPA programı sonuçlarından yaklaşı olarak $1 \mathrm{~K}$ daha yüksek olduğu görülmüştür. Bu değerler dikkate alınmayacak kadar küçük ve önemsiz farklar olarak bilinmektedir.

Tablo 6 RPA-CEA programlarının analiz sonuçlarının karşılaştırılması

\begin{tabular}{lccccc}
\hline & Analiz Programı & Yanma Odası & Nozul Boğazı & Nozul Çıkışı & Birim \\
\hline Basınç & RPA & 20 & 11.26 & 0.1013 & MPa \\
& CEA & 20 & 11.26 & 0.10138 & \\
Sıcaklık & RPA & 3289.88 & 3039.84 & 1309.62 & $\mathrm{~K}$ \\
& CEA & 3290.9 & 3040.94 & 1310.7 & \\
Mak Sayısı & RPA & 0 & 1 & 3.76 & \\
& CEA & 0 & 1 & 3.76 & \\
\hline
\end{tabular}

Tablo 7'de RPA programından alınan emisyon değerleri verilmiştir. Daha önceden belirtildiği üzere maksimum itki kuvveti üretmek için yakıtça zengin karışım oranı seçilmiştir. Analiz sonucuna göre nozul çıkışında serbest oksijen görülmediği için iyi bir yanma elde edilebileceği sonucuna varılabilir. Kullanılan hidrojen ve oksijen yakıt çiftinin yanma sonu ürünü $\mathrm{H}_{2} \mathrm{O}$ olduğundan dolayı çevreye duyarlı, zehirleyici olmayan ve yüksek enerjiye sahip bir yakıt çifti olarak temiz bir enerji kaynağı olduğu görülmektedir.

Tablo 7 RPA programının emisyon sonucu

\begin{tabular}{lccccc}
\hline & Enjektör & Yanma Odası & Nozul girişi & Nozul boğazı & Nozul çıkışı \\
\hline $\mathrm{H}$ & 0 & 0,001369 & 0,0013651 & 0,000911 & 0,0000001 \\
$\mathrm{H}_{2}$ & 0,172413793 & 0,0675675 & 0,0675655 & 0,0675791 & 0,0679398 \\
$\mathrm{H}_{2} \mathrm{O}$ & 0 & 0,9150378 & 0,9151371 & 0,9225336 & 0,9320601 \\
$\mathrm{H}_{2} \mathrm{O}_{2}$ & 0 & 0,0000074 & 0,0000073 & 0,0000025 & 0 \\
$\mathrm{HO}_{2}$ & 0 & 0,0000074 & 0,0000073 & 0,0000021 & 0 \\
$\mathrm{O}$ & 0 & 0,0003207 & 0,0003181 & 0,0001237 & 0 \\
$\mathrm{O}_{2}$ & 0,827586207 & 0,0003779 & 0,000375 & 0,0001491 & 0 \\
$\mathrm{OH}$ & 0 & 0,0153121 & 0,0152246 & 0,0086988 & 0 \\
\hline
\end{tabular}

\section{Tartışma}

Roket performansı artırmak için birçok nozul tasarımı geliştirilmektir. Yapılan araştırmalarda görülmüştür ki; çan tipi nozul genişleme bölgesinin ilk kısmında, hızlı genişlemeye ya da dairesel akışa neden olduğundan nozul çıkışında düzgün ve eksenel akış sağlayacağından dolayı en uygun nozul tipi olarak De laval \%80'lik parabolik yapı seçilmiştir. Çan tipi nozul konik nozul tipine göre daha kısa düşük sürtünme kaybı ve hafif olmaktadır.

Şekil 1'de görüleceği gibi özgül itki; yanma odası sıcaklığı ve gazların molekül ağırlığından doğrudan etkilendiği görülmektedir. Hidrojen ve oksijen yakıt çiftinin stokiyometrik oranı 7.937 olmasına rağmen, en yüksek özgül itki değeri oksitleyici yakıt oranı 4.8 olduğunda elde edilmektedir.

$$
\mathrm{I}_{\mathrm{sp}, \max }=\frac{1}{\mathrm{~g}}\left[\frac{2 \mathrm{k}}{\mathrm{k}-1} \frac{\mathrm{R}}{\mathrm{M}_{\mathrm{g}}} \mathrm{T}_{\mathrm{c}}\left\{1-\left(\frac{\mathrm{P}_{\mathrm{e}}}{\mathrm{P}_{\mathrm{c}}}\right)^{\frac{\mathrm{k}-1}{\mathrm{k}}}\right\}\right]^{\frac{1}{2}}
$$

Denklem 21'de maksimum özgül itki eşitliği görülmektedir (Turner, 2006). 


\section{Sonuç}

Bu çalışmada, öncelikli olarak rokette kullanılacak yakıt ve oksitleyici seçimi yapılmışıı. Yakıt olarak sıvı hidrojen $45 \mathrm{~K}$ ve sıvı oksijen $90 \mathrm{~K}$ sıcaklığı sınır şartlarında kriyojenik olarak rokette kullanılmıştır. Yakıt oksitleyici oranı lisanslı olarak kullanılan RPA programı desteğinde 4.8 olarak ideal oranı belirlenmiştir. Sınır şartları RPA programına girilerek optimum roket geometrisi oluşturulmuştur. Roket analizi girilen $100 \mathrm{kN}$ itki kuvveti üretmesi için yanma odası basınc1 $20 \mathrm{MPa}$ ve dış ortam basınc1 $101.3 \mathrm{kPa}$ olarak belirlenmiştir. Sonrasında roket teorik olarak ateşlenmiş RPA ve CEA programları ile ayrı ayrı analizler gerçekleştirilmiştir. Teorik olarak roket analizleri hızlı bir şekilde gerçekleştirilerek zamandan tasarruf sağlanmaktadır. Yakıt çifti ve karışım oranlarının ve itki odası geometrisinin roket performansı üzerine olan etkisi hızlı bir şekilde incelenebilmektedir. Optimum karışım oranı belirlenebilmektedir. Üretilen gaz hızı, sıcaklığı ve basıncının dağılımı roket boyunca analiz edilmiştir. En yüksek sıcaklık $3290.9 \mathrm{~K}$ ile yanma odasında görülmüştür. Mak sayısı 3.76 çıkmıştır. En yüksek özgül itkinin meydana geldiği 4.8 karışım oranındaki nozul çıkışında kütlesel olarak \%93,20 suya dönüştüğü görülmüştür. Böylece çevreci bir yakıt çifti olduğu değerlendirilmektedir. Bu analizler neticesinde elde edilen sonuçlar değerlendirildiğinde imalatı hedeflenen $100 \mathrm{kN}$ 'luk roketin üretilebilirliği teyit edilmiştir.

Yazarların Katkısı: Tüm çalışmada yazarların test, analiz, literatür araştırması, yazma ve düzeltme işlerinde eşit oranda katkıları olmuştur. Aynı zamanda bu çalışma yüksek lisans tezinden türetilmiştir.

Fon Desteği: RPA program yazarların kendi imkanlarıyla karşılanmıştır. Ayrıca CEA program akademik çalışmalara NASA tarafından açık kaynak olarak ücretsiz bir şekilde sunulmaktadır.

\section{Referanslar}

Baxi, P., Jain, R., Dhadke, Y., Chhabra, Y. ve Khatawate, V. H. (2021). Design and Analysis of Bell-Parabolic De Laval Rocket Exhaust Nozzle. 2021 th Biennial International Conference on Nascent Technologies in Engineering (ICNTE) içinde (ss. 1-6). IEEE.

Cai, G., Fang, J., Xu, X. ve Liu, M. (2007). Performance Prediction nd Optimization for Liquid Rocket Engine Nozzle. Aerospace Science and Technology, 11(2-3), 155-162.

Cengiz, K. (2010). Development Of An Iterative Method For Liquid-Propellant Combustion Chamber Instability Analysis. Uzay Mühendisliği. Orta Doğu Teknik Üniversitesi.

Dönmez, C. E. (2018). Kompozİt Esasli Kati Roket Yakit Numunesİİn Karaktersittík Özellíklerİİ́n Deneysel İncelenmesi. FEN BILIMLERI ENSTITÜSÜ. Kırıkkale Üniversitesi.

Gill, G. S. ve Nurick, W. H. (1976). Liquid Rocket Engine Injectors.

Hetem, A., Miraglia, J., Burian, R. ve Caetano, C. A. C. (2011). Numerical Simulation Of Liquid Propellant Rocket Engines. MIPRO, 2011 Proceedings of the 34th International Convention içinde (ss. 930-934). IEEE.

Huang, D. H. ve Huzel, D. K. (1971). Design of Liquid Propellant Rocket Engines Second Edition.

Karayel, E. (2018). Roket Motor Borusu Tasarımının Balistik Parametrelere Etkisinin Araştırılması. fen Bilimleri Enstitüsü. Kırıkkale Üniversitesi.

Ngwu, G. O., Ugheoke, B. I., Yusuf, O. T., Nyabam, M. A. ve Onuh, S. O. (2020). Numerical Analysis and Modelling of a $100 \mathrm{~N}$ Hypergolic Liquid Bipropellant Thruster. Advances in Aerospace Science and Technology, 5(4), 85-99.

Ojeda, C., Prescott, K. T. ve Persaud, T. (2017). Production and Manufacture of Low-Cost Liquid Rocket Engines for Sounding Rockets. 53rd AIAA/SAE/ASEE Joint Propulsion Conference içinde (s. 4841).

Pillai, E. T. (2018). Space-capable sounding rocket design for collegiate teams. 2018 IEEE Aerospace Conference içinde (ss. 1-13). IEEE.

Ponomarenko, A. (2009). RPA: Design tool for liquid rocket engine analysis.

Raposo, H. C. T. (2016). Mixture Ratio and Thrust Control of a Liquid-Propellant Rocket Engine. Aerospace Engineering. Centre National D'études Spatiales. 
Rodríguez Otero, R., Morales-Jimenez, S., Ureña Contreras, K. ve Baez Cortes, J. (2020). Loxodon-1: Development of the First Liquid Rocket Engine in Puerto Rico. ASCEND 2020 içinde (s. 4258).

Samur, A. E. (2015). Hİbrìt Yakitli Roket Motoru Ateşleme/Test Düzeneğ̇̇ Tasarimi. Havacilik Ve Uzay Teknolojillerİ Enstittüsü. Hava Harp Okulu.

Santos, E. A., Alves, W. F., Prado, A. N. A. ve Martins, C. A. (2011). Development of Test Stand For Experimental Investigation of Chemical and Physical Phenomena in Liquid Rocket Engine. Journal of Aerospace Technology and Management, 3(2), $159-170$. doi:10.5028/jatm.2011.03021111

Strunz, R. (1998). Using The Method Of Characteristics To Predict Transient Flow Phenomena.

Sutton, G. P. ve Biblarz, O. (2016). Rocket propulsion elements. John Wiley \& Sons.

Türk, S. (2016). Üst Kademe Sıvı Yakıtlı Roket Motoru İçin Kavramsal İtki Odası Tasarımı. Fen Bilimleri Enstitüsü. Anadolu Üniversitesi, Eskişehir.

Turner, M. J. L. (2006). Rocket and Spacecraft Propulsion (2. bs.). Springer-Verlag Berlin Heidelberg.

Ward, T. A. (2010). Aerospace propulsion systems. John Wiley \& Sons.

Yaman, H. (2013). Yüksek Enerjili Maddelerin Çift Bazlı (DB) Roket Yakıtlarında Kullanımı ve Performans Etkilerinin Araştırılması. Fen Bilimleri Enstitüsü. Kırıkkale Üniversitesi, Kırıkale.

Youngblood, S. H. (2015). Design and Testing of a Liquid Nitrous Oxide and Ethanol Fueled Rocket Engine. New Mexico Institute of Mining and Technology, Department of Mechanical Engineering, Explosive Engineering. 